\title{
Molecular Dynamics Simulation of Electrons in Gold Nanoparticles
}

\author{
M. SALANDARI ${ }^{a, *}$ AND H. SABZYAN ${ }^{b}$ \\ ${ }^{a}$ Department of Chemistry, Payame Noor University, P.O. Box 19395-3697, Tehran, Iran \\ ${ }^{b}$ Department of Chemistry, University of Isfahan, Isfahan 81746-73441, Islamic Republic of Iran
}

(Received February 1, 2020; in final form February 24, 2020)

\begin{abstract}
Electron dynamics in gold $\left(\mathrm{Au}_{n}\right)$ nanoparticles in the absence and presence of electric field is studied using molecular dynamics simulations. For this dynamics, motion of electrons is treated classically by considering a force field including Coulomb and van der Waals interaction potentials for the electron-electron $\left(\mathrm{e}^{-}-\mathrm{e}^{-}\right)$, electron-ionic core $\left(\mathrm{e}^{-}-\mathrm{Au}^{+}\right)$and ionic core-ionic core $\left(\mathrm{Au}^{+}-\mathrm{Au}^{+}\right)$pairs of species. These electrons and ionic cores are set initially at the alternative sites of a set of identical twin fcc lattices. Electric current and conduction are evaluated for the $\mathrm{Au}_{n}$ gold nanoparticles of different sizes $(n=2048,2816,3328$, and 3840) in electric field of $0.001 \mathrm{~V} / \AA$ strength. Results of this study show a non-linear dependence of electric conduction on the size of the gold nanoparticles.
\end{abstract}

DOI: 10.12693/APhysPolA.137.1175

PACS/topics: electron dynamics, gold nanoparticles, molecular dynamic simulation, electric conductivity

\section{Introduction}

Electron transfer (ET) is one of the most important elementary processes in chemistry. An elementary ET reaction is characterized by the transfer of at least one electron from one species to another, or from one part of a molecule to the other without any change in the bonding connectivity in the contributing species and molecules. The ET reaction, thus, appears as a change in the electron density distribution over the reacting species [1]. ET reactions occurring in the bulk of liquid and at the solid-liquid interface have wide applications in chemical, electrochemical, biological and semiconductors processes and especially in photovoltaic solar cells [2]. It is thus necessary to study ET at atomic/molecular level in order to understand mechanisms of these processes and improve performance of the electronic devices and photovoltaic solar cells. Furthermore, pure physical ET in which no chemical reaction is involved, play a central role as precursor of reactive ET processes and in the performances of solid state devices in the form of displacement of electrons in an electrical conductance or across an electronic junction, as well as in all electrostatic phenomena which have both useful and destructive effects $[3,4]$ in many industrial processes. Specifically, production of electrostatic charges and its consequent physical ET contribute both constructively and destructively in the production, processing and applications of powders of different materials, especially nanoparticles (NPs). Therefore, detailed knowledge of ET is crucial in modifying and improving processes involved in the production and manipulation of NPs.

\footnotetext{
*e-mail: msalandari85@yahoo.com
}

Nanoparticles have 1-100 nm sizes [5-7]. One significant consequence of this length scale is a high surface area to volume ratio that results in unique properties such as high catalytic activities and specific quantum effects including non-ohmic and discrete electric conductance. Quantum behavior of electrons in discrete pathways on the surface or in the bulk of nanosize or nanostructure materials can enhance or inhibit electric conduction of these materials. Size, geometry and bonding configuration of NPs determine this quantum behavior [8].

Metal nanoparticles are of special importance due to the ease of production with the desired sizes and shapes, and with controlled electric, magnetic, optical, chemical and physical properties including high electrical conduction $[9,10]$. The metallic nanoparticles with high electric conductance can be applied as a thin layer in solar cells [11]. Because of chemical stability and resistance to corrosion, good alloying properties and high electric conductance, gold NPs have found wider applications in the study and production of nano-electronic devices $[12,13]$ working based on the quantum transfer of electrons.

The bulk gold crystals adopt a face-centered cubic (fcc) crystal structure, while gold NPs may have crystallized or amorphous structures and appear in different shapes and sizes [14]. Gold NPs have fascinating characteristics such as low-symmetry structures at some geometric magic numbers [15] and size-related electronic, magnetic [16] and optical properties [17] due to quantum relativistic and size effects. Some atomic and macroscopic properties of gold are listed in Table I.

Molecular dynamics simulation (MDS) is carried out to understand behavior and evaluate properties of assemblies of molecules in terms of their composition, structure and microscopic interactions. Results of MDSs serve as a complement for the results of conventional experimental measurements, and to establish a connection between the microscopic length/time scales characteristics and macroscopic properties and behaviors. The choice 
TABLE I

Some atomic and macroscopic properties of gold $(\mathrm{Au})$

\begin{tabular}{l|c|c}
\hline \hline \multicolumn{1}{c|}{ Property } & Value & Ref. \\
\hline atomic number & 79 & {$[18]$} \\
atomic weight & $196.967 \mathrm{~g} / \mathrm{mol}$ & {$[18]$} \\
isotope (abundance) & ${ }^{197} \mathrm{Au}(100 \%)$ & {$[19]$} \\
melting point & $1064^{\circ} \mathrm{C}$ & {$[19]$} \\
crystal structure & fcc & {$[20]$} \\
unit cell parameter & $4.078 \AA$ & {$[20]$} \\
density & $19.3 \mathrm{~g} / \mathrm{cm}^{3}$ & {$[15]$} \\
electrical conductivity $\left(20{ }^{\circ} \mathrm{C}\right)$ & $4.1 \times 10^{7} \mathrm{~S} / \mathrm{m}$ & {$[15]$} \\
electrical resistivity $\left(20^{\circ} \mathrm{C}\right)$ & $2.44 \times 10^{-8} \Omega \mathrm{m}$ & {$[15]$}
\end{tabular}

of an accurate and descriptive potential energy function is crucial to derive reliable and accurate results in MDS studies [21].

The ultimate aim of this series of studies is to model electron dynamics including electric conduction (EC) and electron transfer (ET) processes in metal NPs under external electric field (voltage) within MDS approaches. Gold nanoparticles are considered as prototype, specifically because of reported well-developed synthetic approaches and characterization techniques [13, 17, 18].

Presently, no well-defined force field is available for the description of the motion of electrons and its consequent properties such as electric conductance. This is due to the quantum behavior of electrons at atomic and molecular length scales and significantly smaller mass of electron compared to the mass of atoms by factors of $\approx 1837 \mathrm{~A}$ (with $\mathrm{A}$ being the mass number of the involved atoms) which requires simulations with reduced time scales, at least four orders of magnitude smaller than those used for most MDSs. For MDS of the motion of electrons, thus, much larger number of steps (by the same factor) is needed, as compared to those of ordinary atomistic MDSs.

\section{Computation model and details}

The electron dynamics/transfer (conduction) process is modeled by considering the gold nanoparticles $\left(\mathrm{Au}_{n}\right.$ $\mathrm{NPs}$ ) to be composed of interacting gold ionic cores $\left(\mathrm{Au}^{+}\right)$and electrons $\left(\mathrm{e}^{-}\right)$. The ionic cores and electrons are set at the sites of a twin fcc lattice having two sublattices, one for ionic cores and the other for electrons, both with fcc mutually intercalated structures and $a=b=c=4.078 \AA$ cubic unit cell dimensions, which are shifted in space by $\left(\frac{1}{2} a, \frac{1}{2} b, \frac{1}{2} c\right)$ along the $(x, y, z)$ axes, with respect to each other. In this model, the ionic cores and electrons are thus set alternatively in space as shown in Fig. 1. The supercells of our MDS have identical dimensions $\left(l_{x} \times l_{y}\right)=(30.585 \times 30.585) \AA^{2}$ along the $x$ and $y$ axes, but with different dimensions $l_{z}=30.585,42.819$, 50.975 , and $59.131 \AA$ along the $z$-axis, which are produced by repeating the twin unit cells along the three orthogonal axes. These supercells thus contain 2048, 2816, 3328 , and 3840 gold atoms, respectively.

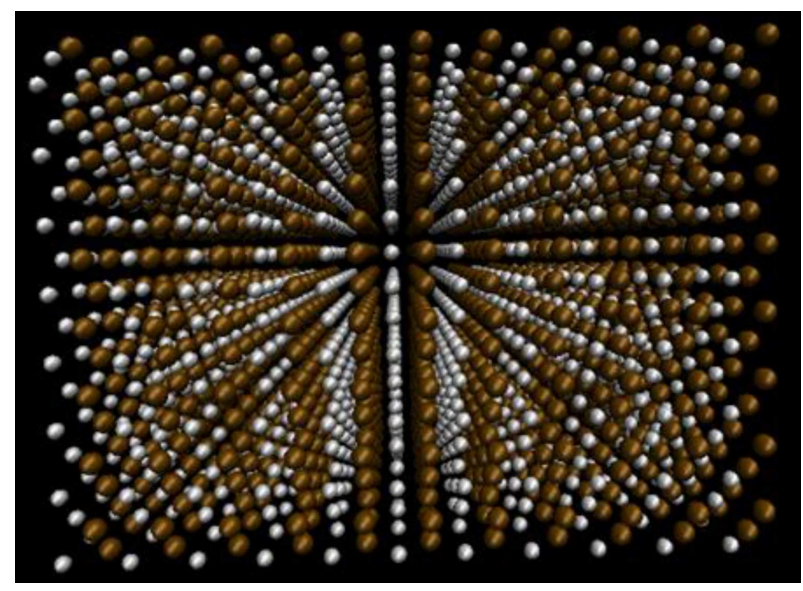

Fig. 1. An example of the initial geometry of a rectangular gold nanoparticle modeled by a set of two identical mutually intercalated (twin) fcc lattices of $\mathrm{Au}^{+}$(brown balls) $+\mathrm{e}^{-}$(white balls)] particles, used in this study for the simulation of the electron dynamics in gold nanoparticles.

In these MDSs, canonical ensemble with constant number of particles, volume and temperature (abbreviated as NVT) is applied without periodic boundary conditions at temperature $T=85 \mathrm{~K}$ (corresponding to the normal boiling point of argon) using the Brensden thermostat with a $\tau=0.0005$ ps relaxation time. The step time and total number of steps are $1 \times 10^{-6}$ ps and $1 \times 10^{7}$, respectively. Complete charges of $+e$ and $-e$ are assigned to the gold ionic cores and electrons, i.e. $\mathrm{Au}^{+}$and $\mathrm{e}^{-}$, respectively. All present MDSs are carried out using LAMMPS software $[22,23]$.

In addition to the Coulomb electrostatic interactions, non-electrostatic Lennard-Jones potentials are also used for better description of the gold-gold $\left(\mathrm{Au}^{+}-\mathrm{Au}^{+}\right)$, goldelectron $\left(\mathrm{Au}^{+}-\mathrm{e}^{-}\right)$and electron-electron $\left(\mathrm{e}^{-}-\mathrm{e}^{-}\right)$interactions accounting partially for the non-classical behavior of electrons in this system. The Lennard-Jones cutoff radii are considered to be $3 \sigma$, but the cutoff distances of the Coulomb interactions are set equal to the largest dimension of the simulation box. In these MDSs carried out for the simulation of the electron dynamics in gold NPs, no periodic boundary condition is applied so that issues related to minimum image convention and saw-dent potential do not arise.

\subsection{Lennard-Jones potential parameters ( $\sigma$ and $\varepsilon$ )}

Inclusion of the Lennard-Jones van der Waals potential for the interactions including electrons and gold ionic cores is based on the fact that the Coulomb interaction cannot describe properly the non-local and extended nature of electrons, especially at very short and very long inter-particle distances. The pure electrostatic interactions result in strong attraction of oppositely charged particles which should be avoided. Furthermore, strong Coulomb repulsion of identically charged particles results in their ejection from the NPs (especially for 
electrons due to their significantly smaller masses), and thus should be suppressed. Therefore, inclusion of van der Waals type of interaction is necessary in the modeling of the dynamics of electrons, as it partially corrects unrealistic behavior of pairwise interaction potentials at the limiting distances. To represent the quantum nature of electrons perfectly, more complex potentials are needed whose development is not intended in this step of our studies.

Atomization energy of the bulk solid gold (368.40 $\mathrm{kJ} / \mathrm{mol} \mathrm{[24]),} \mathrm{corresponding} \mathrm{to} \mathrm{an} \mathrm{Au}-\mathrm{Au}$ bond energy of $61.4 \mathrm{~kJ} / \mathrm{mol}$ in the fcc crystal structure having coordination number 12 , and the work function of the gold NPs $(3.6 \mathrm{eV}[25])$ are used to estimate the $\varepsilon$ and $\sigma$ parameters for the $\mathrm{Au}^{+}-\mathrm{Au}^{+}$and $\mathrm{Au}^{+}-\mathrm{e}^{-}$pair interactions.

Since electrons are very lighter than the gold ionic cores, the spatial moves (jumps) predicted and applied in each step of the molecular dynamics simulation may be so large to result in superposition with each other and with the gold ionic cores in some instances of simulation. Therefore, small values of collision diameter $\left(\sigma_{\mathrm{e}^{-}-\mathrm{e}^{-}}\right)$ should be considered for the $\mathrm{e}^{-}-\mathrm{e}^{-}$Lennard-Jones interaction potential.

Based on the criteria and estimations described above, several sets of $(\varepsilon, \sigma)$ values, listed in Table II, are considered and NVT MDSs are carried out, and their corresponding lattice binding energies $E_{b}$ and work functions $W$ are calculated and analyzed. As can be seen in Table II, for some sets of $(\varepsilon, \sigma)$ values, the gold NP system is not even stable and undergoes decomposition. The best results are obtained with the $0.15 \mathrm{eV}$ and $0.08 \mathrm{eV}$ values for the Lennard-Jones interaction potential well depths $\varepsilon_{\mathrm{Au}^{+}-\mathrm{Au}^{+}}$and $\varepsilon_{\mathrm{Au}^{+-\mathrm{e}^{-}}}$, respectively.

To evaluate performance of the force field parameters, the gold nanoparticle work function $W$ corresponding to the $\mathrm{NP} \rightarrow \mathrm{NP}^{+}+\mathrm{e}^{-}$ionization reaction and binding energy $\left(E_{b}\right)$ corresponding to the energy change of the $\mathrm{NP}\left(\mathrm{Au}_{n}\right) \rightarrow \mathrm{NP}^{\prime}\left(\mathrm{Au}_{\mathrm{n}-1}\right)+\mathrm{Au}$ reaction are considered as two objective and reference characteristics to be reproduced by present MDSs. For the calculation of the work function $W$, one electron is removed from the lattice, and the whole simulation is carried out under the same conditions. The energy difference $E\left(\mathrm{NP}^{+}\right)-E(\mathrm{NP})$ is then considered to be equal to $W$. Also, for the calculation of the gold binding energy $E_{b}$, MDS is carried out for the $\mathrm{Au}_{n}$ and $\mathrm{Au}_{n-1}$ NPs, and the energy difference $E\left(A u_{n}\right)-E\left(A u_{n-1}\right)$ is considered to be equal to $E_{b}$. A review of these results, reported in Table II shows that the values of the two characteristics (work function and binding energy) are very sensitive to the variations of the force field parameters. Also, the best simultaneous agreement between the calculated values obtained for both binding energy and work function quantities in the present work and the corresponding experimental values $[24,25]$ is obtained only with the force field parameter set No. 14. With this set of force field parameter values, the temperature fluctuations are settled down better within $\pm 4 \mathrm{~K}$ (at $T=85 \mathrm{~K}$ ). Therefore, in the rest part of this study, this set of values is used for the simulation of electron dynamics/conduction in the gold NPs under external electric field.

TABLE II

Stability of the molecular dynamics simulations carried out on the $\mathrm{Au}_{2048}$ cubic gold nanoparticle using different sets of values for the $\mathrm{Au}^{+}-\mathrm{Au}^{+}, \mathrm{Au}^{+}-\mathrm{e}^{-}$and $\mathrm{e}^{-}-\mathrm{e}^{-}$van der Waals interaction force field parameters. In these simulations, NVT ensemble at $T=85 \mathrm{~K}$ (normal boiling point of argon) with the Brensden thermostat $(\tau=0.0005$ ps relaxation time) are used. The total number of steps and step time are $1 \times 10^{7}$ and $1 \times 10^{-6} \mathrm{ps}$, respectively. The values of $\varepsilon$ and $\sigma$ are given in $\mathrm{eV}$ and $\AA$, respectively. The binding energy $\left(E_{b}\right.$ in $\left.\mathrm{eV}\right)$ and work function ( $W$ in $\left.\mathrm{eV}\right)$ obtained for this gold $\mathrm{NP}$ are also reported in the last two columns. Experimental values reported for the binding energy and work function of gold are $E_{b}=0.64 \mathrm{eV}[24]$ and $W=3.6 \mathrm{eV}$ [25], respectively.

\begin{tabular}{c|c|c|c|c|c|c|c|c|c|c}
\hline \hline No. & $\varepsilon_{\mathrm{Au}^{+}-\mathrm{Au}^{+}}$ & $\varepsilon_{\mathrm{Au}^{+}-\mathrm{e}^{-}}$ & $\varepsilon_{\mathrm{e}^{-}-\mathrm{e}^{-}}$ & $\sigma_{\mathrm{Au}^{+}-\mathrm{Au}^{+}}$ & $\sigma_{\mathrm{Au}^{+}-\mathrm{e}^{-}}$ & $\sigma_{\mathrm{e}^{-}-\mathrm{e}^{-}}$ & $T[\mathrm{~K}]$ & $E_{b}$ & $W$ & System stability \\
\hline 1 & $0.46^{a}$ & 0.03 & 0.050 & $3.64^{a}$ & 1.5 & 0.05 & 591 & 2.64 & 17.35 & unstable \\
2 & 0.64 & 0.36 & 0.001 & 2.90 & 1.9 & 1.0 & 485 & 2.84 & 108.3 & unstable \\
3 & 0.64 & 0.05 & 0.001 & 2.56 & 2.1 & 1.1 & 162 & 1.50 & 101.6 & unstable \\
4 & 0.40 & 0.30 & 0.001 & 2.56 & 2.1 & 0.9 & 314 & 1.44 & 11.65 & unstable \\
5 & 0.30 & 0.10 & 0.005 & 2.56 & 2.1 & 0.9 & 348 & 0.78 & 5.63 & unstable \\
6 & 0.13 & 0.08 & 0.001 & 2.56 & 1.9 & 0.9 & 97 & 0.72 & 1.02 & stable \\
7 & 0.12 & 0.07 & 0.005 & 2.56 & 1.9 & 1.0 & 95 & 0.65 & 0.36 & stable \\
8 & 0.12 & 0.06 & 0.001 & 2.56 & 1.9 & 0.9 & 94 & 0.59 & 1.38 & stable \\
9 & 0.11 & 0.06 & 0.001 & 2.56 & 1.9 & 0.9 & 86 & 0.56 & 0.23 & stable \\
10 & 0.11 & 0.05 & 0.005 & 2.56 & 2.1 & 1.0 & 85 & 0.52 & 5.01 & stable \\
11 & 0.10 & 0.05 & 0.001 & 2.56 & 1.9 & 0.9 & 87 & 0.29 & 0.44 & stable \\
12 & 0.10 & 0.06 & 0.005 & 2.56 & 1.8 & 0.9 & 88 & 0.29 & 0.43 & stable \\
13 & 0.15 & 0.08 & 0.001 & 2.56 & 1.9 & 0.4 & 160 & 0.72 & 4.86 & stable \\
14 & 0.15 & 0.08 & 0.001 & 2.56 & 1.9 & 0.9 & 82 & 0.70 & 3.27 & stable \\
\hline
\end{tabular}

${ }^{a} \operatorname{Ref}[26]$ 

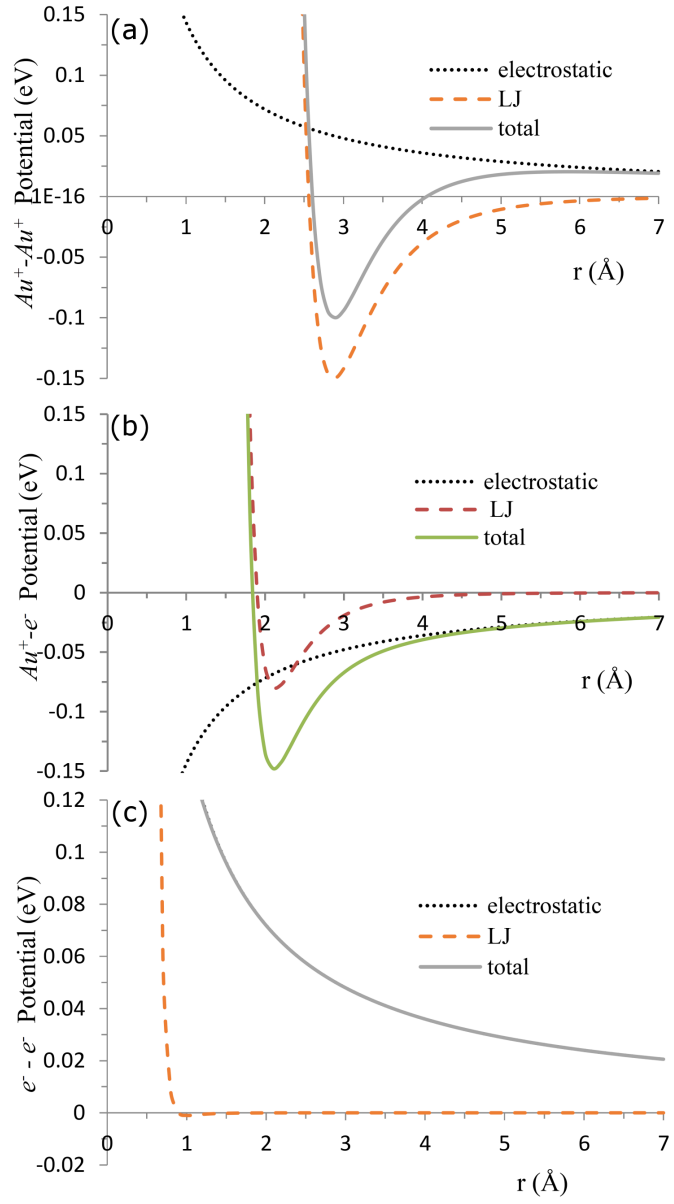

Fig. 2. Electrostatic, van der Waals (Lennard-Jones, LJ) and total pair potential energy functions for the $\mathrm{Au}^{+}-\mathrm{Au}^{+}$(a), $\mathrm{Au}^{+}-\mathrm{e}^{-}$(b), and $\mathrm{e}^{-}-\mathrm{e}^{-}$(c) interactions corresponding to the parameter set No. 14 listed in Table II. Note that the electrostatic component and the total interaction potential are superimposed over all $\mathrm{e}^{-}-\mathrm{e}^{-}$distance ranges shown in this figure.

The total potential energy curves corresponding to the optimized values of force field parameters (set No. 14 in Table II) are plotted in Fig. 2. As can be seen in this figure, for this set of force field parameter values, in the $\mathrm{Au}^{+}-\mathrm{Au}^{+}$and $\mathrm{Au}^{+}-\mathrm{e}^{-}$interactions, the LJ potential is predominant, while in the $\mathrm{e}^{-}-\mathrm{e}^{-}$interaction, the electrostatic potential is predominant. As is expected naturally, the $\mathrm{e}^{-}-\mathrm{e}^{-}$interaction potential is repulsive over whole range of the $\mathrm{e}^{-}-\mathrm{e}^{-}$distances. Also, addition of van der Waals interaction makes all three total potentials much harder at short distances.

\subsection{Electron transfer in gold nanoparticles under electric field}

In order to study electric conductivity of the gold NPs, molecular dynamics simulations are carried out also in the presence of external electric field of different strengths in the range of $(2-10) \times 10^{-4} \mathrm{~V} / \AA$ applied along the $+z$-direction of the simulation box. Stronger
TABLE III

Effects of electric fields of different strengths on the binding energy $E_{b}$ and work function $W$ of the cubic $\left(\mathrm{Au}_{2048}\right)$ gold nanoparticle obtained at the end of $1 \times 10^{7}$ step NVT $(T=85 \mathrm{~K})$ MDSs using the force field parameters set No. 14 introduced in Table II. Average equilibrium temperature $T_{\text {ave }}$ and its fluctuation $\Delta T$ are also reported.

\begin{tabular}{c|c|c|c|c}
\hline \hline $\begin{array}{c}\text { Electrical field } \\
{\left[10^{-4} \mathrm{~V} / \AA\right]}\end{array}$ & $T_{\text {ave }}[\mathrm{K}]$ & $\Delta T[\mathrm{~K}]$ & $E_{b}[\mathrm{eV}]$ & $W[\mathrm{eV}]$ \\
\hline 10 & 86 & 3.9 & 0.63 & 3.74 \\
8 & 87 & 3.9 & 0.61 & 2.56 \\
6 & 81 & 4.0 & 0.61 & 1.61 \\
4 & 80 & 3.9 & 0.60 & 2.04 \\
2 & 83 & 4.0 & 0.60 & 3.43 \\
0 & 82 & 1.8 & 0.70 & 3.27
\end{tabular}

electric fields are also examined, but they resulted in instabilities and ionization of the NPs. Furthermore, no significant or observable electric displacement (current) is produced by electric fields weaker than $2 \times 10^{-4} \mathrm{~V} / \AA$. Values of binding energies obtained in the MDSs in the presence of electric field are listed in Table III. For the study of electron dynamics and the effect of the length of the super cell, only electric field of $0.001 \mathrm{~V} / \AA$ strength is considered in this report. Behavior of electrons in these simulations is characterized and described in the next section.

\section{Results and discussion}

\subsection{Radial distribution function}

Distributions of the gold core ions $\mathrm{Au}^{+}$and the electrons $\mathrm{e}^{-}$over the simulation box are characterized by calculating and analyzing the radial distribution function (RDF) for the $\mathrm{Au}^{+}-\mathrm{Au}^{+}, \mathrm{Au}^{+}-\mathrm{e}^{-}$, and $\mathrm{e}^{-}-\mathrm{e}^{-}$pairs. Typical RDF curves calculated for the geometry of the $\mathrm{Au}_{2048}$ gold nanoparticle in the absence of electric field in the first and last steps of an extra $5 \times 10^{5}$ step MDS, and in the last step of the $1 \times 10^{7}$ MDS in the presence of electric field of $0.001 \mathrm{~V} / \AA$ strength are plotted respectively in parts (a), (b) and (c) of Fig. 3. It can be seen from Fig. 3a that even in the first step of simulation, electrons are randomly displaced significantly such that the structure of the $\mathrm{e}^{-}-\mathrm{e}^{-}$RDF disappears immediately at the beginning of the simulation. This is while the well-structured $\mathrm{Au}^{+}-\mathrm{e}^{-} \mathrm{RDF}$ shows that the electrons adopt a relatively structured distribution around the gold ionic cores. On the other hand, low peak height observed for the $\mathrm{e}^{-}-\mathrm{e}^{-}$RDF indicates that the electrons are not accumulated but rather distributed over the crystal space among the gold ionic cores. Also, the $\mathrm{Au}^{+}-\mathrm{Au}^{+}$ and $\mathrm{Au}^{+}-\mathrm{e}^{-} \mathrm{RDF}$ peaks in the absence and presence of electric field of $0.001 \mathrm{~V} / \AA$ show that the gold ionic cores are also displaced, but with much smaller scales as compared to those of the $\mathrm{e}^{-}-\mathrm{e}^{-}$RDF. The calculated RDF at $t=0.05 \mathrm{ps}$ in the absence of electric field plotted 

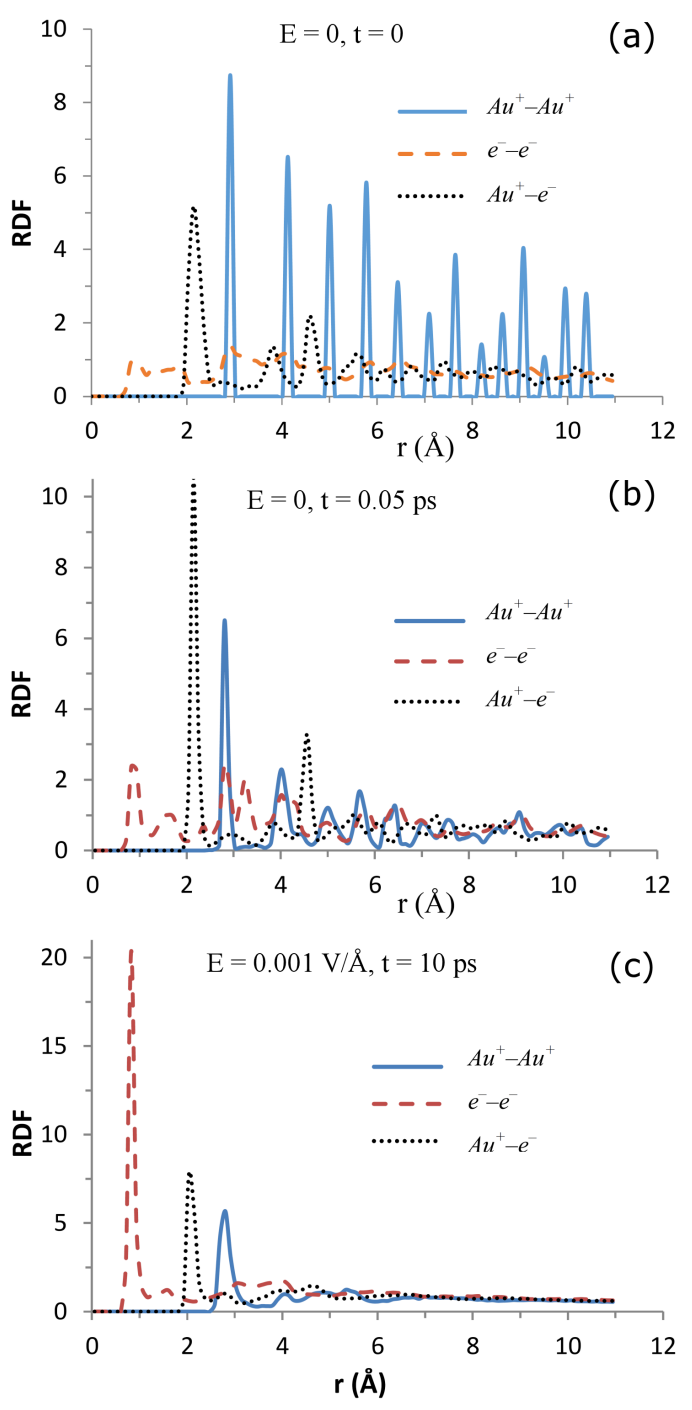

Fig. 3. Radial distribution functions (RDF) calculated for the $\mathrm{Au}^{+}-\mathrm{Au}^{+}, \mathrm{Au}^{+}-\mathrm{e}^{-}$, and $\mathrm{e}^{-}-\mathrm{e}^{-}$pairs in the $\mathrm{Au}_{2048}$ gold NP in the NVT simulations described in the text with the force field parameter set No. 14 (Table II), in the first (a) and the last (b) steps of simulation in the absence of electric field, and (c) in the last step of the $1 \times 10^{7}$ step simulation in the presence of electric field of $0.001 \mathrm{~V} / \AA$ strength.

in Fig. 3b display more or less uniform distribution of electrons over the space of the NP. When electric field is applied, electrons are partially concentrated in one side of the NP and thus the first peak of the $\mathrm{e}^{-}-\mathrm{e}^{-}$RDF becomes more intense (Fig. 3c). The features of the RDF curves described above mean that the values of force field parameters are set properly and can be used for simulation of the electron dynamic/conductance process of our interest.

\subsection{Displacement of the electrons}

The root of mean square displacements (RMSD) of the electrons along the three Cartesian coordinates of the NP
TABLE IV

The root of mean square displacements (RMSDs) of electrons obtained for the $1 \times 10^{7}$ step NVT MDSs carried out on the cubic $\mathrm{Au}_{2048}$ gold NP with the force field parameter sets 2, 5 and 14 (Table II) in the absence and presence of electric field of $0.001 \mathrm{~V} / \AA$ strength along the $+z$-axis.

\begin{tabular}{c|c|c|c|c|c|c}
\hline \hline \multirow{2}{*}{ No. } & \multicolumn{6}{|c}{ RMSD } \\
\cline { 2 - 7 } & \multicolumn{3}{|c|}{$E=0$} & \multicolumn{3}{|c}{$E=0.001 \mathrm{~V} / \AA$} \\
\cline { 2 - 7 } & $x$ & $y$ & $z$ & $x$ & $y$ & $z$ \\
\hline 2 & 0.4 & 0.4 & 0.4 & 1.6 & 1.7 & 1.7 \\
5 & 0.4 & 0.4 & 0.3 & 1.6 & 1.7 & 2.7 \\
14 & 0.2 & 0.2 & 0.3 & 1.7 & 1.8 & 3.0
\end{tabular}

at the final step of simulations are calculated for the force field parameter set 14 (Table II) both in the absence and in the presence of electric field of $0.001 \mathrm{~V} / \AA$ strength. These calculated RMSDs are reported in Table IV where results obtained with the parameter sets 2 and 5 (Table II) are also given for comparison. As can be seen in this table, the least electron displacements at zero field belongs to the force field parameters set No. 14, which is selected as the best (optimized) set. Also, the RMSD values along all three directions, especially the direction of the applied electric field (i.e. $+z$ ) increases as compared to that in the absence of electric field. These increased RMSDs can be attributed to the enhanced collisions of electrons as a result of their partial concentration along the $+z$-direction due to the applied electric field. Exceptional increase in the RMSD of the electrons in the $z$ direction, as an obvious result of the applied electric field, denotes also the conduction of electrons in this direction.

\subsection{Electric polarization}

Electric polarization, i.e. dipole moment, is induced in an object when an electric field distorts its uniform electric charge distribution. In our systems of the $\mathrm{Au}_{n}$ gold NPs, the applied electric field displaces the electrons inside the relatively fixed lattice of the gold ionic cores, thus, separating the centers of the negative (electrons) and positive (gold ionic cores) charges. The electric polarization $\boldsymbol{P}$ (with the components $p_{x}, p_{y}$, and $p_{z}$ ) induced by this separation is calculated at a limited number of points over the course of the NVT simulation on the $\mathrm{Au}_{2048}$ gold NP, and plotted in Fig. 4. As can be seen in this figure, the polarization in the $x$ - and $y$-directions (i.e. $p_{x}$ and $p_{y}$ ) are very small, because the electric field has no component along these two directions. The initial polarizations along all three directions are due actually to the unavoidable offset arising from initial setup of the shifted twin lattices (alternate positions of the electrons and gold ionic cores) which does not allow superposition of their centers (origins) to give expected zero polarization at the initial time.

It can also be seen from Fig. 4 that the induced charge polarization along the $z$-axis $\left(p_{z}\right)$ increases at the early and intermediate stages of the simulation, but fluctuates 


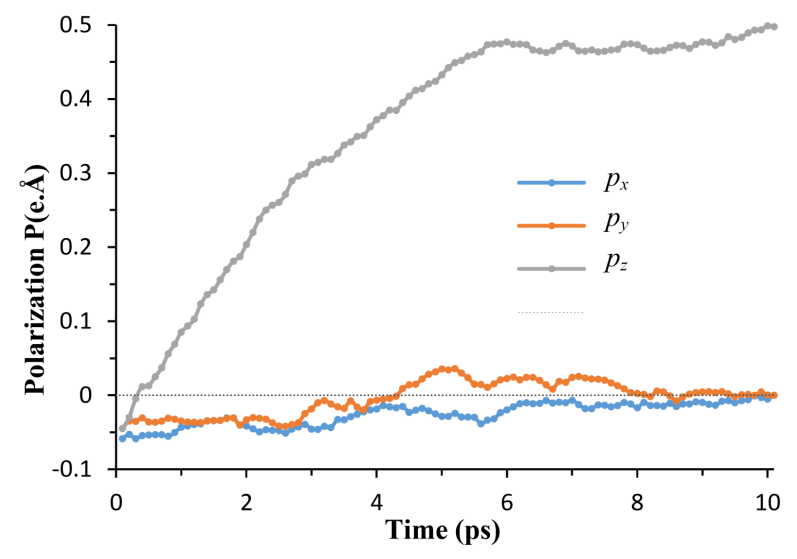

Fig. 4. Electric charge polarization induced in the $\mathrm{Au}_{2048}$ gold nanoparticle during the course of a $1 \times 10^{7}$ step NVT MDS in the presence of the electric field of $0.001 \mathrm{~V} / \AA$ applied along the $+z$-direction.

without significant change towards an asymptotic value in the late stage of the simulation. This behavior, which can be attributed to the accumulation of electrons and their increased repulsion opposing the effect of the electric field, is typical of the non-infinite systems such as nanosized particles in our case.

\subsection{Electric current and electric conductivity}

The main purpose of this series of works is to evaluate electrical conductivity of gold nanoparticles created by the motion of electrons in response to an applied electric potential. Electrical conductivity measures the ability of a medium (its charge carriers) to move under applied electric field [27, 28]. When electrical field $\boldsymbol{E}$ is turned on, motion of electrons along the field direction produces an electrical current $\boldsymbol{I}$ parallel to the direction of the electric field $\boldsymbol{E}$, which is given by

$$
I=N^{*} e A_{\perp} v_{z},
$$

where $N^{*}$ is the number density of the charge carriers (electrons here), $e$ is the charge of electron, $A_{\perp}$ is the surface area of the cross-section perpendicular to the direction of the electric current produced by electrons of velocity $v_{z}$. The electric conductance $(\sigma)$ of the medium under electric field of $E_{z}$ strength is thus evaluated using

$$
\sigma=\frac{J_{z}}{E_{z}}, \quad J_{z}=\frac{I_{z}}{A_{\perp}} .
$$

$J_{z}$ is the magnitude of the current density.

The average electron velocity $v_{z}$, its consequent electric current $I_{z}$, and electric conductance $\sigma$ are calculated for the simulated motion of electrons in the gold NPs of different sizes (fixed $l_{x}$ and $l_{y}$, but different $l_{z}$ dimensions) in an $0.001 \mathrm{~V} / \AA$ electric field applied along the $+z$-direction. Results of these calculations are reported in Table V. Variation of the calculated electrical conductivities of these gold NPs are also presented pictorially in Fig. 5.

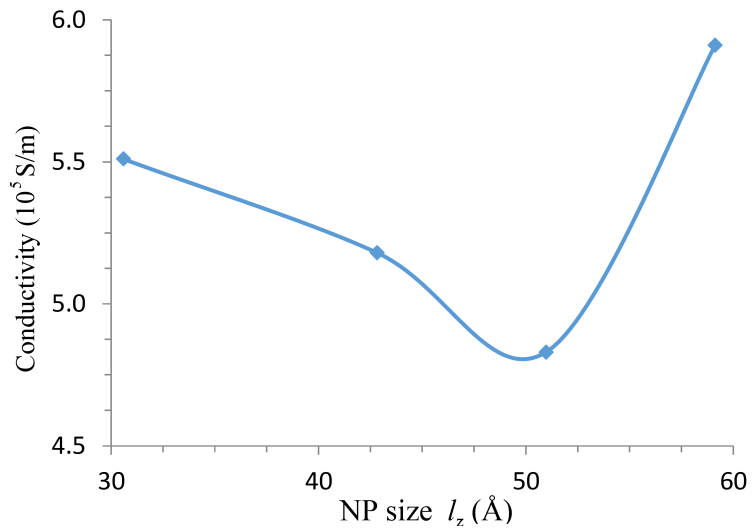

Fig. 5. Dependence of the electron conductivity on the size $l_{z}$ of the $\mathrm{Au}_{n}$ gold nanoparticle.

TABLE V

Calculated values of velocities $v_{z}$, electric current $I_{z}$, and conductance $\sigma$ of electrons obtained for the rectangular $\mathrm{Au}_{n}$ NPs based on the NVT simulations under electric field of $0.001 \mathrm{~V} / \AA$ strength showing the effect of the NPs size in the $z$-direction $l_{z}$. The experimental value of conductivity for the bulk gold (i.e. of macroscopic sizes) is reported to be $4.42 \times 10^{7} \mathrm{~S} / \mathrm{m}[15]$.

\begin{tabular}{c|c|c|c|c}
\hline \hline $\mathrm{NP}$ & Size $l_{z}[\AA]$ & $v_{z}[\mathrm{~m} / \mathrm{s}]$ & $I_{z}\left[10^{5} \mathrm{~A}\right]$ & $\sigma\left[10^{5} \mathrm{~S} / \mathrm{m}\right]$ \\
\hline $\mathrm{Au}_{2048}$ & 30.6 & 960 & 5.14 & 5.51 \\
$\mathrm{Au}_{2816}$ & 42.8 & 946 & 4.86 & 5.18 \\
$\mathrm{Au}_{3328}$ & 51.0 & 910 & 4.52 & 4.83 \\
$\mathrm{Au}_{3840}$ & 59.1 & 1065 & 5.53 & 5.91
\end{tabular}

Analysis of the data reported in Table $\mathrm{V}$ shows that the calculated conductivity first decreases and then increases with increasing the gold NPs size $l_{z}$. Since, to our knowledge, no experimental or computational (simulation) value is so far reported for the gold nanoparticles, evaluation of the present simulation cannot be accomplished.

\section{Conclusion}

Molecular dynamic simulations (MDSs) are carried out to study electron dynamics in gold nanoparticles ( $\mathrm{Au}$ NPs) in the absence and presence of electric field. The motion of electrons in these NPs is modelled by placing initially the gold ionic cores $\left(\mathrm{Au}^{+}\right)$and electrons $\left(\mathrm{e}^{-}\right)$on the sites of a fcc twin crystal lattice corresponding to the experimental crystal of the gold. The Au NPs considered in our studies have rectangular shapes with identical sizes along the $x$ - and $y$-axes, but different sizes along the $z$-axis containing 2048, 2816, 3328, and 3840 atoms. Molecular dynamic simulations are carried out within NVT ensemble at $T=85 \mathrm{~K}$ using the Brendsen thermostat of $t=0.0005$ ps relaxation time. A total number of $1 \times 10^{7}$ steps with the time-step of $1 \times 10^{-6}$ ps are used in all simulations. Values of the force field parameters are optimized towards a stable lattice throughout 
the course of molecular dynamics simulations and reproduction of the experimentally reported gold lattice work function and bonding energy. Radial distribution functions calculated for the simulated nanoparticles approve the set of values adopted for the force field parameters. Finally, electric field is applied along the $+z$-direction, and the electrical conductivity of gold NPs is calculated based on the displacement of electrons under electric field of $0.001 \mathrm{~V} / \AA$ strength. Results of this study show nonlinear dependence of the conductivity of the gold NPs on its size along the $z$-axis. The calculated conductances of the gold NPs are smaller than that experimentally reported for the bulk macroscopic gold which suggests development of more accurate interaction potentials, especially for the electrons.

\section{References}

[1] D. Small, D.V. Matyushov, G.A. Voth, J. Am. Chem. Soc. 125, 7470 (2003).

[2] J.R. Bolton, N. Mataga, G. Mc Lendon, Advances in Chemistry Series 228, Ed. J.R. Bolton, N. Mataga, G. McLendon, American Chemical Society, Washington 1991.

[3] C.E. Lapple, Adv. Chem. Eng. 8, 1 (1970).

[4] K.S. Jamieson, H.M. ApSimon, J.N. Bell, J. Phys. Conf. Series 142, 012052 (2008).

[5] R. Singh, D. Pantarotto, D. Mc Carthy et al., J. Am. Chem. Soc. 127, 4388 (2005).

[6] R. Nagarajan, T.A. Hatton, Nanoparticles: Synthesis, Stabilization, Passivation, and Functionalization, Oxford University Press, Washington 2008.

[7] F. Baletto, R. Ferrando, Rev. Mod. Phys. 77, 371 (2005).

[8] M.-C. Daniel, D. Astruc, Chem. Rev. 104, 293 (2004).

[9] P.A. Montano, G.K. Shenoy, E.E. Alp, W. Schulze, J. Urban, Phys. Rev. Lett. 56, 2076 (1986).

[10] Sh. Chen, J. Mater. Chem. 17, 4115 (2007).

[11] B. Niesen, N. Blondiaux, M. Boccard et al., Nano Lett. 14, 5085 (2014).
[12] L. Janovák, I. Dékány, J. Appl. Surf. Sci. 256, 2809 (2010).

[13] G. Yang, L. Hu, T.D. Keiper, P. Xiong, D.T. Hallinan, Langmuir 32, 4022 (2016).

[14] J. Meija, T.B. Coplen, M. Berglund et al., Pure Appl. Chem. 88, 265 (2016).

[15] D. Levesque, J.J. Weis, L. Reatto, Phys. Rev. Lett. 56, 1212 (1986).

[16] D.B. Leznoff, B.Y. Xue, R.J. Batchelor, F.W.B. Einstein, B.O. Patrick, Inorg. Chem. 40, 6026 (2001).

[17] J.M. Pomeroy, H. Grube, A.C. Perrella, J.D. Gillaspy, Phys. Rev. B 75, 241409(R) (2007).

[18] W. Huang, M. Ji, Ch.-D. Dong, X. Gu, L.M. Wang, X.G. Gong, L.S. Wang, ACS Nano 2, 897 (2008).

[19] P. Crespo, R. Litrán, T.C. Rojas et al., Phys. Rev. Lett. 93, 087204 (2004).

[20] A.L. González, C. Noguez, A.S. Barnard, J. Phys. Chem. C 116, 14170 (2012)

[21] J. Schmidt, J. Vande-Vondele, I.-F.W. Kuo, D. Sebastiani, I. Siepmann, J. Hutter, C.J. Mundy, J. Phys. Chem. B 113, 11959 (2009).

[22] A. Dequidt, J. Devémy, A.A.H. Pádua, J. Chem. Inf. Model. 56, 260 (2016).

[23] LAMMPS Molecular Dynamic Simulation, Sandia National Laboratories, 2010; S. Plimpton, J. Comp. Phys. 117, 1 (1995).

[24] J.W. Arblaster, J. Phase Equilib. Diff. 37, 229 (2016)

[25] Y. Zhang, O. Pluchery, L. Caillard, A.-F. LamicHumblot, S. Casale, Y.J. Chabal, M. Salmeron, Nano Lett. 15, 51 (2015).

[26] A.A. Tseng, in: Tip-Based Nanofabrication: Fundamentals and Applications, Ed. A.A. Tseng Springer, New York 2011, p. 401.

[27] A. J. Dekker, Solid State Physics, Macmillan, London 1981, p. 275.

[28] S. Kasap, C. Koughia, H.E. Ruda, in: Springer Handbook of Electronic and Photonic Materials, Eds. S. Kasap, P. Capper, Springer, Cham 2017, p. 19. 